\title{
Multiple-ancestry genome-wide association study identifies 27 loci associated with measures of hemolysis following blood storage
}

Grier P. Page, ${ }^{1}$ Tamir Kanias, ${ }^{2}$ Yuelong J. Guo, ${ }^{3}$ Marion C. Lanteri, ${ }^{4}$ Xu Zhang,,${ }^{5}$ Alan E. Mast, ${ }^{6}$ Ritchard G. Cable, ${ }^{7}$ Bryan R. Spencer, ${ }^{8}$ Joseph E. Kiss, ${ }^{9}$ Fang Fang, ${ }^{3}$ Stacy M. Endres-Dighe, ${ }^{10}$ Donald Brambilla, ${ }^{10}$ Mehdi Nouraie, ${ }^{11}$ Victor R. Gordeuk, ${ }^{5}$ Steve Kleinman, ${ }^{12}$ Michael P. Busch, ${ }^{4}$ Mark T. Gladwin, ${ }^{13,14}$ and the National Heart, Lung, and Blood Institute (NHLBI) Recipient Epidemiology Donor Evaluation Study-III (REDS-III) program ${ }^{15}$

'Division of Biostatistics and Epidemiology, RTI International, Atlanta, Georgia, USA. ${ }^{2}$ Vitalant Research Institute, Denver, Colorado, USA. ${ }^{3}$ Division of Biostatistics and Epidemiology, RTI International, Durham, North Carolina, USA. ${ }^{4}$ Vitalant Research Institute and the Department of Laboratory Medicine, UCSF, San Francisco, California, USA. ${ }^{5}$ Department of Medicine, University of Illinois at Chicago, Chicago, Illinois, USA. ${ }^{6}$ Blood Research Institute, Blood Center of Wisconsin, and Department of Cell Biology, Neurobiology and Anatomy, Medical College of Wisconsin, Milwaukee, Wisconsin, USA. ${ }^{7}$ American Red Cross, Farmington, Connecticut, USA. ${ }^{8}$ American Red Cross, Dedham, Massachusetts, USA. ${ }^{9}$ Vitalant Northeast Division, Pittsburgh, Pennsylvania, USA. ${ }^{10}$ Division of Biostatistics and Epidemiology, RTI International, Rockville, Maryland, USA. "Division of Pulmonary, Allergy and Critical Care Medicine, Department of Medicine, University of Pittsburgh, Pennsylvania, USA. ${ }^{2}$ University of British Columbia, Victoria, British Columbia, Canada. ${ }^{3}$ Pittsburgh Heart, Lung, and Blood Vascular Medicine Institute, University of Pittsburgh, Pittsburgh, Pennsylvania, USA. ${ }^{14}$ Division of Pulmonary, Allergy and Critical Care Medicine, Department of Medicine, University of Pittsburgh Medical Center, Pittsburgh, Pennsylvania, USA. ${ }^{15}$ The NHLBI REDS-III program is detailed in the Supplemental Acknowledgments.

Background. The evolutionary pressure of endemic malaria and other erythrocytic pathogens has shaped variation in genes encoding erythrocyte structural and functional proteins, influencing responses to hemolytic stress during transfusion and disease.

Methods. We sought to identify such genetic variants in blood donors by conducting a genome-wide association study (CWAS) of 12,353 volunteer donors, including 1,406 African Americans, 1,306 Asians, and 945 Hispanics, whose stored erythrocytes were characterized by quantitative assays of in vitro osmotic, oxidative, and cold-storage hemolysis.

Results. CWAS revealed 27 significant loci $\left(P<5 \times 10^{-8}\right)$, many in candidate genes known to modulate erythrocyte structure, metabolism, and ion channels, including SPTA1, ALDH2, ANK1, HK1, MAPKAPK5, AQP1, PIEZ01, and SLC4A1/band 3. CWAS of oxidative hemolysis identified variants in genes encoding antioxidant enzymes, including GLRX, GPX4, G6PD, and SEC14L4 (Golgi-transport protein). Genome-wide significant loci were also tested for association with the severity of steady-state (baseline) in vivo hemolytic anemia in patients with sickle cell disease, with confirmation of identified SNPs in HBA2, G6PD, PIEZO1, AOP1, and SEC14L4.

Conclusions. Many of the identified variants, such as those in G6PD, have previously been shown to impair erythrocyte recovery after transfusion, associate with anemia, or cause rare Mendelian human hemolytic diseases. Candidate SNPs in these genes, especially in polygenic combinations, may affect RBC recovery after transfusion and modulate disease severity in hemolytic diseases, such as sickle cell disease and malaria.

\section{Introduction}

Blood transfusion is one of the most common procedures during hospital stays, with more than 36,000 red blood cell (RBC) trans-

Authorship note: GPP and TK are co-first authors. MPB and MTC are co-senior authors.

Conflict of interest: MB declares NHLBI REDS salary support. MTC declares industry consulting relationships with Pfizer, Bayer, Fulcrum, and Novartis. VG declares institutional grants or contracts from CSL Behring, Novartis, Global Blood Therapeutics, Imara, and Ironwood, and data and safety monitoring board or advisory board participation with CSL Behring, Novartis, Global Blood Therapeutics, and Forma. Copyright: @ 2021, American Society for Clinical Investigation.

Submitted: November 13, 2020; Accepted: May 13, 2021; Published: July 1, 2021

Reference information: J Clin Invest. 2021;131(13):e146077.

https://doi.org/10.1172/JCl146077. fusions performed daily in the United States. Clinically, RBC transfusions are largely considered to be homogeneous. However, a growing number of studies have evaluated the potential impact of unique donor characteristics, such as sex, age, and body mass index, on RBC storage integrity (1-3), posttransfusion recovery and survival of RBCs, and consequent clinical outcomes (4-7). In addition, the US donor population is ethnically diverse, with hundreds of functionally and immunologically relevant RBC single-nucleotide polymorphisms (SNPs) $(8,9)$.

Studies evaluating inbred mouse strains demonstrated strong heritable determinants of RBC susceptibility to canonical in vitro stressors such as cold-storage hemolysis, osmotic hemolysis, and oxidative hemolysis; importantly, these in vitro responses also correlated with posttransfusion $\mathrm{RBC}$ recovery and function $(4,10,11)$. 
In humans, in vitro hemolysis of donor RBCs in response to osmotic or oxidative stress is a reproducible and heritable trait that can be further modulated by factors such as donation history, ancestry, age, and sex $(12,13)$. Human studies of RBC recovery and survival following blood bank storage have demonstrated variability among donors that is reproducible over time, suggesting donor-specific factors such as sickle cell trait (7) and glucose-6-phosphate dehydrogenase (G6PD) deficiency (14-16), can reduce posttransfusion $\mathrm{RBC}$ recovery (17). Indeed, in a recent study, the posttransfusion $\mathrm{RBC}$ recovery was evaluated in 10 volunteers with G6PD deficiency using chromium-51 cell labeling. Recovery was $78.5 \%$ in G6PD-deficient subjects versus $85.3 \%$ for transfusion in 27 control subjects without G6PD ( $P=0.0009$; ref. 16$)$.

Genetic variability also contributes to the intensity of hemolysis observed in Mendelian hemolytic diseases, such as SCD. In patients who are homozygous for the hemoglobin S variant allele (HbS), there is significant variability in the intensity of steady-state or baseline hemolysis (18-21). Coinheritance of $\alpha$ - and $\beta$-thalassemia and mutations modulating the expression of fetal hemoglobin ( $\mathrm{HbF})$ influence hemoglobin levels and hemolysis in patients with SCD $(21,22)$. Furthermore, variability in severity of hemolysis influences clinical outcomes (23), promoting vasculopathy and the development of end-organ complications, such as pulmonary hypertension, cutaneous leg ulceration, and chronic kidney injury. We and others have demonstrated that cell-free hemoglobin released during hemolysis in the setting of SCD and transfusion of aged, stored blood is toxic, driving nitric oxide depletion, oxidative injury, heme-mediated inflammation, and iron overload (19-21, 23, 24).

These findings inform the hypothesis that rare and common genetic variants modulate various characteristics of erythrocytes leading to altered susceptibility to hemolysis that may influence erythrocyte storage in blood banks, transfusion outcomes, and potentially the severity of hemolytic diseases. Considering this hypothesis, the aim of this study was to identify genes that modulate hemolysis in cold storage and hemolytic disorders by conducting a genome-wide association (GWA) study in RBC donors enrolled in the National Heart, Lung, and Blood Institute (NHLBI) $\mathrm{RBC}$-Omics project $(2,25)$. We tested the associations between in vitro measures of stress hemolysis in cold-stored RBCs (spontaneous storage hemolysis, osmotic fragility, and oxidative hemolysis) and high-density GWA SNPs (26) to discover candidate loci that regulate the function of human RBCs and their resilience to stress. This GWA cohort of 12,353 volunteer donors was enriched for groups with African, Hispanic, and Asian ancestry. Collected and stored RBCs were characterized by quantitative assays for in vitro osmotic, oxidative, and cold-storage hemolysis. Consistent with the anticipated genetic variability in donor RBCs, our analysis identified 27 GWA-significant loci $\left(P<5 \times 10^{-8}\right)$, many in candidate genes known to modulate erythrocyte structure, metabolism, and ion channels. We further verified whether the SNPs identified from our in vitro hemolytic stress phenotyping have relevance to hemolytic disease by analyzing their association with in vivo measurements of the severity of steady-state (outpatient) hemolytic anemia (low hemoglobin values and high indices of hemolysis) in 2 cohorts of SCD patients.

These studies suggest that the identification of genetic variables that modulate the stability of RBCs in storage, response to stressors, and the functional integrity of RBCs after transfusion could advance donor selection criteria and procedures and storage policies. Identification and removal of genetically susceptible RBC donors/units that rapidly degrade in storage (exclusion of "fragile" RBC donors/units) and selection of profiled "super donors" that might be stable for longer periods of storage or survive longer after transfusion could provide for a precision transfusion medicine strategy, more advanced than current random sampling of donors and transfusion of RBC units irrespective of recipient disease status or short- or long-term transfusion requirements. In addition, the variants could provide information about risk and severity of hemolytic anemia in patients with hemolytic diseases, such as SCD, thalassemia, and malaria, as well as advance the discovery of proteins and enzymes that modulate RBC function.

\section{Results}

Population ancestry of REDS-III RBC-Omics cohort. The RBC-Omics cohort included a diverse group of US blood donors born in many $(n=71)$ countries. Initially, groups were divided into continental ancestry groups; however, we have followed recent recommendations to divide the Hispanic $(27,28)$ and Asian ancestry groups into multiple subgroups based on country of birth. Donors of Hispanic ancestry were divided into 2 groups: Mexican and Central American Hispanics (MCAH) (Supplemental Figures 1 and 2; supplemental material available online with this article; https://doi.org/10.1172/JCI146077DS1) and Caribbean Island Hispanics (CIH) (Supplemental Figures 1 and 3). Donors of Asian ancestry were divided into East Asians and South Asians to reflect the diversity of these RBC-Omics subpopulations (27, 28). In total, the REDS-III RBC-Omics populations (Figure 1A) were divided into 7 ancestry groups that included non-Hispanic Whites $(n=7,586)$, East Asians $(n=1,049)$, South Asians $(n=257)$, MCAH $(n=456)$, CIH $(n=489)$, African Americans $(n=1,046)$, and "Other" participants $(n=1,336)$. "Other" participants is a heterogeneous group including all individuals that did not cluster within the other groups, but included people who self-identified as Native Americans, Native Hawaiians, Native Alaskans, multiple races, or were from countries like Iran and the Philippines. We also considered the entire RBC-Omics as a single group referred to as ALL Ancestries.

GWA studies of osmotic, oxidative, and storage hemolysis in mega-analysis. The SNP-based heritability from linkage disequilibrium (LD) score regression for osmotic hemolysis was 0.348 (SEM $=0.062)$, and for oxidative hemolysis was $0.156(\mathrm{SEM}=0.073)$. The heritability score for storage hemolysis was not different from zero. Genome-wide analysis of 12,353 subjects from the REDS-III RBC-Omics cohort was conducted between 14.1 million genotyped and imputed SNPs for osmotic (Figure 1B), oxidative (Figure 1C), and cold-storage hemolysis (Figure 1D). GWA analyses using ALL Ancestries samples identified 14, 4, and 2 genome-wide significant regions that were associated with osmotic, oxidative, and spontaneous cold-storage hemolysis, respectively (Table 1). Q-Q plots (Supplemental Figure 4) did not exhibit any $P$-value inflation.

Genome-wide analysis of osmotic hemolysis in the entire data set (ALL Ancestries) revealed that the genome-wide significant variants were in or close to several logical candidate genes known to modulate RBC structure and function, such as spectrin 

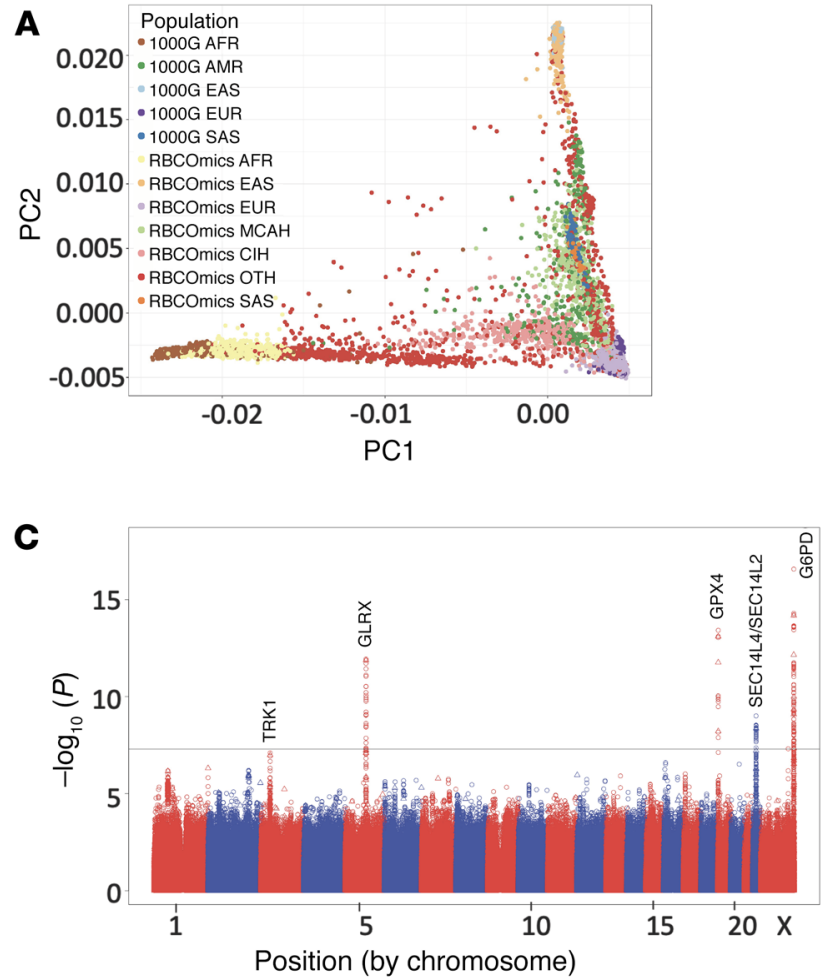
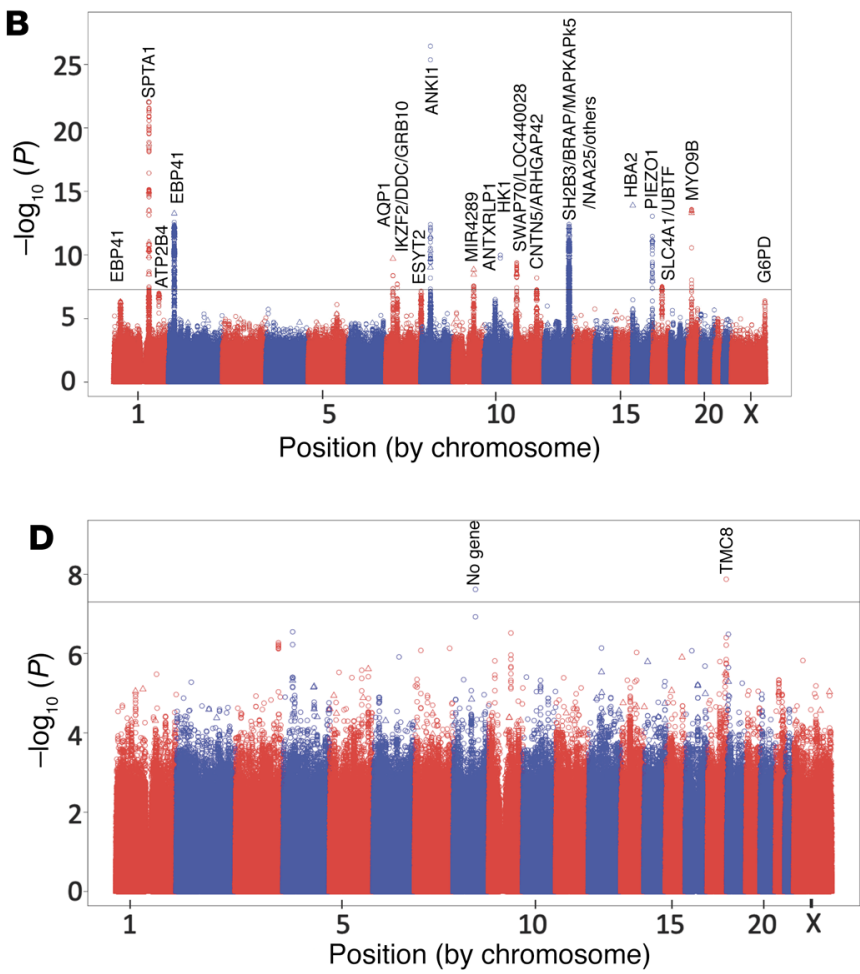

Figure 1. Ancestry of RBC-Omics population and Manhattan plots. (A) Plot of the first 2 principal components (PCs) of the extended RBC-Omics population overlaid on the 1000 Genomes phase 3 samples. Individuals are labeled by genetic ancestry (AFR, African American; EAS, East Asian; SAS, South Asian; EUR, non-Hispanic White; AMR, admixed American; CIH, Caribbean Island Hispanics; MCAH, Mexican and Central American Hispanics; OTH, other/ multiple ancestry) overlain by ancestry groups from 1000 Genomes v3. (B-D) Manhattan plots summarizing the mega-analysis results for osmotic hemolysis $(n=12,215, \lambda=1.003$; B), oxidative hemolysis $(n=10,007, \lambda=1.048$; C), and storage hemolysis $(n=12,177, \lambda=1.002$; $\mathbf{D})$. Each data point corresponds to a $-\log _{10}(P$ value $)$ from a multivariant linear regression model's $P$ value for an SNP. The black horizontal line represents an accepted $P$-value level of genomewide significance $\left(P=5 \times 10^{-8}\right)$. Circles represent noncoding variants, and triangles are coding variants.

$\alpha$ chain, erythrocytic 1 (SPTA1/band $\left.1 ; P<1.01 \times 10^{-22}\right)$, ankyrin 1 (ANK1/band 2.1; $\left.P<5.85 \times 10^{-28}\right)$, aquaporin 1 ( $A Q P 1 ; P<4.23 \times$ $10^{-10}$ ), and solute carrier family 4 member 1 (SLC4A1/band 3; $P$ $\left.<3.62 \times 10^{-8}\right)$ (Table 1). In addition, a number of potentially novel GWA-significant variations were found in metabolic enzymes (hexokinase $1[H K 1] ; P<4.90 \times 10^{-11}$ ), stress kinases (MAPKAPK5; $P<2.24 \times 10^{-13}$ ), ion channels (piezo-type mechanosensitive ion channel component 1 [PIEZO1]; $\left.P<4.04 \times 10^{-14}\right)$, and other proteins, such as myosin IXB $\left(M Y O 9 B ; P<9.88 \times 10^{-15}\right)$. Supporting the internal validity of these findings, many of these SNPs are in proteins known to cause RBC disorders such as spherocytosis (23), elliptocytosis (29), xerocytosis (30), and $\alpha$-thalassemia (31).

GWA analysis of oxidative hemolysis identified genome-wide significant SNPs in G6PD $\left(P<2.66 \times 10^{-17}\right)$, SEC14-like 4 (SEC14L4; $\left.P<9.85 \times 10^{-10}\right)$, glutaredoxin $\left(G L R X ; P<1.15 \times 10^{-12}\right)$, and glutathione peroxidase 4 (GPX4; $\left.P<3.80 \times 10^{-14}\right)$. G6PD, GLRX, and GPX 4 are all known to have roles in protecting cells from oxidative damage. Analysis of storage hemolysis (Figure 1D) identified only 2 genome-wide significant loci: one on chromosome 8 more than $500 \mathrm{~kb}$ from the nearest genes, and another on chromosome 17 (TMC $\left.8 ; P<1.34 \times 10^{-8}\right)$.

Ancestry-specific GWA results. Individual principal component analysis-defined (PCA-defined) ancestry-group GWA revealed a high degree of overlap with the ALL Ancestries analysis; however,
7 additional genome-wide significant loci were observed in genes such as EYS $\left(P<3.20 \times 10^{-9}\right), H B B\left(P<3.66 \times 10^{-10}\right), H B A 2(P<$ $\left.2.90 \times 10^{-14}\right)$, and $G 6 P D\left(P<2.66 \times 10^{-17}\right)$ within specific ancestry groups (Table 1 ) and in only some cases (G6PD and HBA2) were the results significant in the ALL Ancestries analysis. Several loci such as GPX4 and SEC14L4 were only significant when considered with ALL Ancestries groups together. Only studying hemolysis in ancestry-specific analysis and in combined analysis enabled the discovery of all 27 of these loci.

Identification and bioinformatics analysis of variation. We identified 12 directly genotyped genome-wide significant $\left(P<5 \times 10^{-8}\right)$ nonsynonymous variants (NSVs) for hemolysis measures in the entire population or in the ancestry-specific groups, predicted using SIFT (https://sift.bii.a-star.edu.sg/) or PolyPhen2 (http:// genetics.bwh.harvard.edu/pph2/). SPTA1 contains the NSV rs857725 (Lys1693Gln, $P<8.75 \times 10^{-21}$; Figure 2A). Notably, the marker for $\alpha$-thalassemia (Figure 2B) deletion (chr16: 223678) and the $\mathrm{HbS}$ variant modulated osmotic, oxidative, and spontaneous storage hemolysis (7). In $H B B$, the HbS variant (rs334, Glu7Val) was significantly associated $\left(P<3.66 \times 10^{-10}\right)$ with osmotic hemolysis in the African American ancestry group (Figure 2C). For oxidative hemolysis, SEC14L4 AX-83171224/rs9606739 (Arg124Gly, $P<3.07 \times 10^{-9}$; Figure 2D) and G6PD rs1050828 (Val68Met, $P<$ $2.66 \times 10^{-17}$; Figure $2 \mathrm{E}$ ) were significant NSVs, whereas for sponta- 


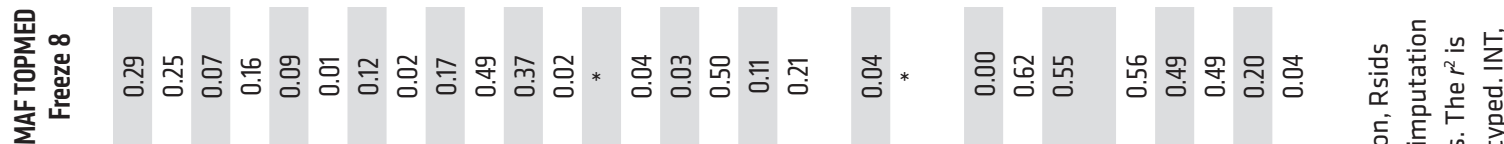

憘

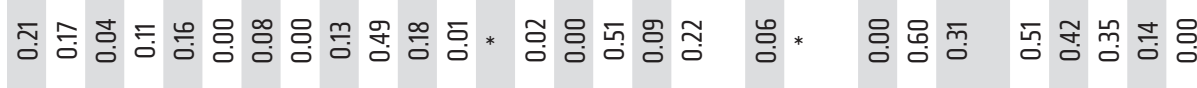

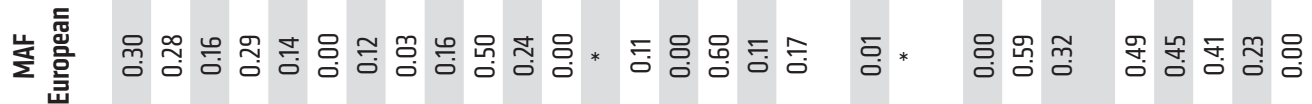

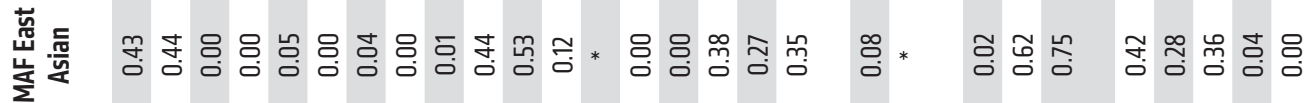

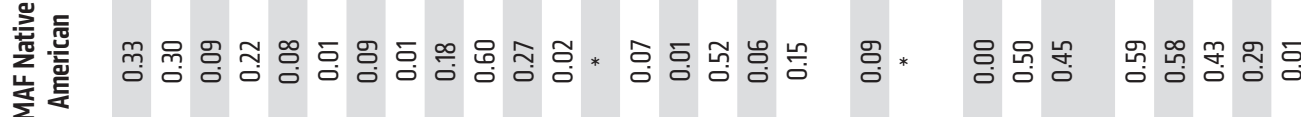

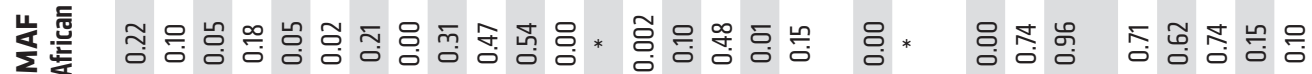

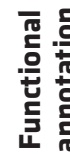

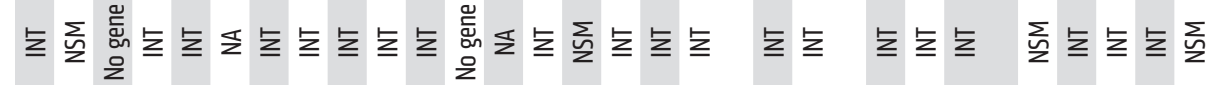

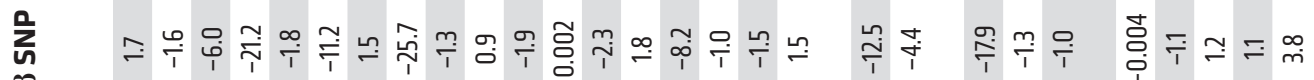

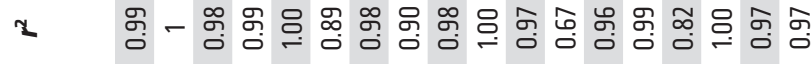

홀

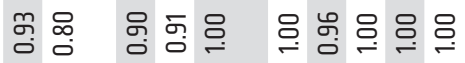
$-\frac{\bar{d}}{\frac{1}{8}}$ $\frac{\overline{\mathrm{g}}}{\dot{\mathrm{d}}} \cup$

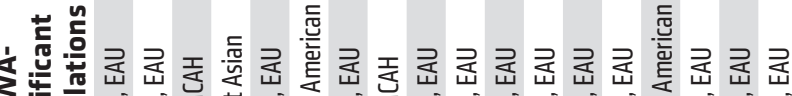

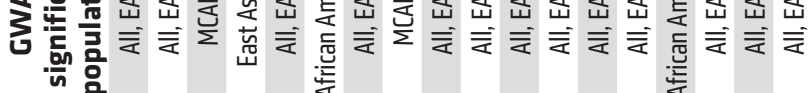

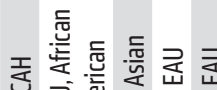

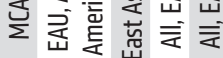
示

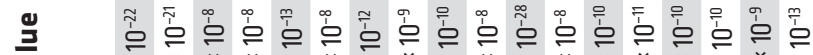

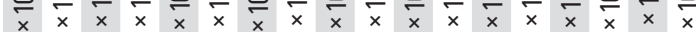

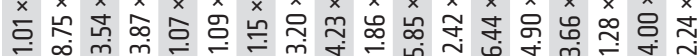

응

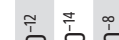
$x \times \frac{x}{x} \times$

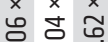

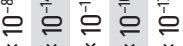
宽品 要

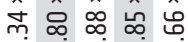

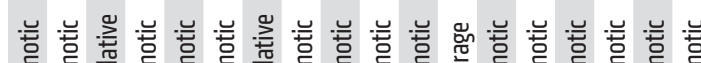

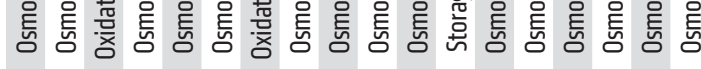

늠

产 咅

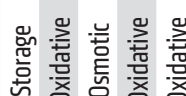

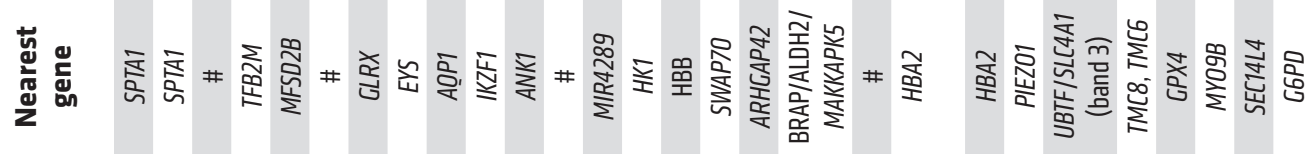

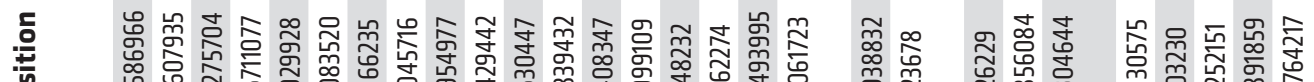

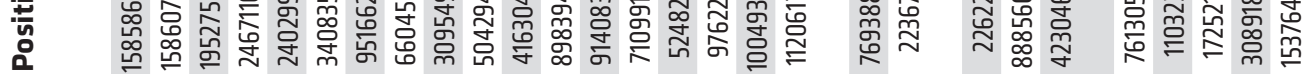

을 吾

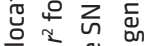

ه

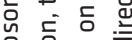

空

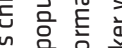

乌응 훈

苞言

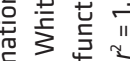

结

元

产亭员

崩京这

일 on

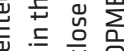

这要号

닌

突总

政

总进范

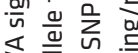

政

品.

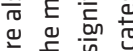

更志

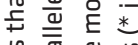

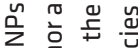

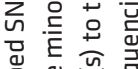

造㐘

힝ㅎㅁ

के

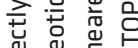

言 造

远宁可

听

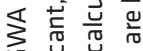

讨

离总

党言

品品品

约元。

苞芹喜

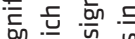

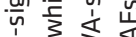

药. $\sum_{0} \sum$

ह ज्ञ

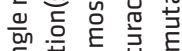

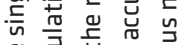

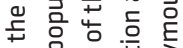

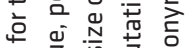

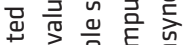
항 흘

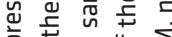

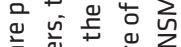

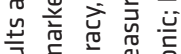

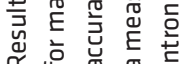


A

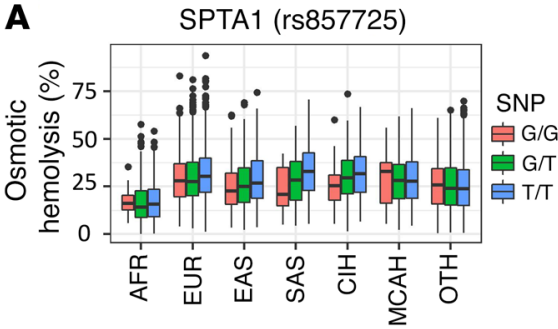

SEC14L4

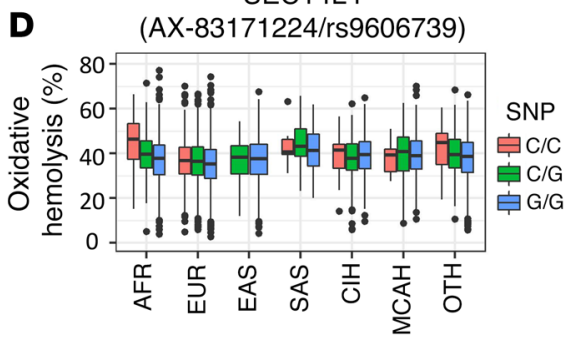

B
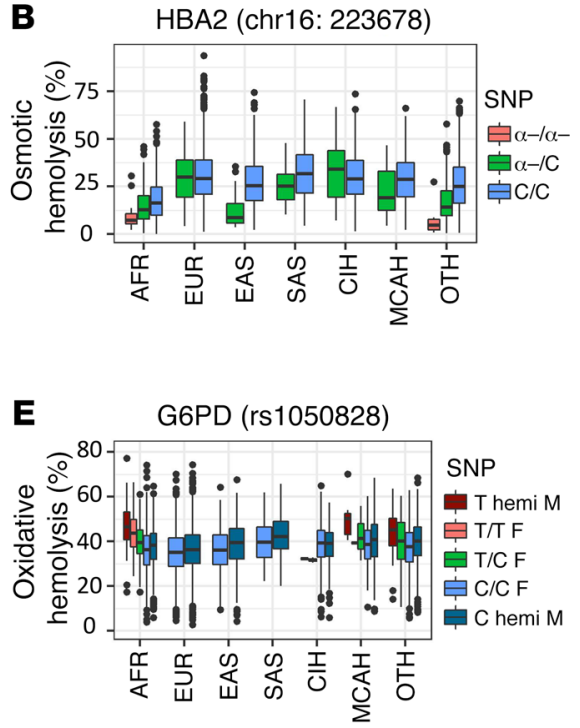

C

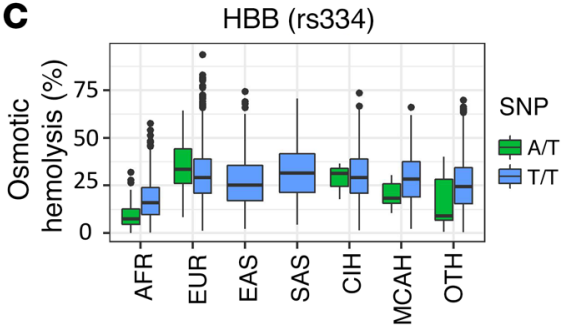

$\mathbf{F}$

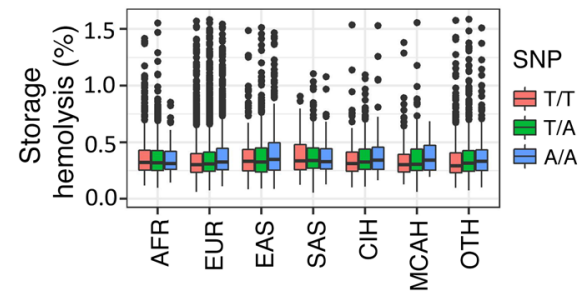

Figure 2. Box-and-whisker plots of various hemolysis levels by genotype for GWA-significant nonsynonymous variants by ancestry group. Osmotic hemolysis: (A) Osmotic SPTA1 (rs857725/Lys1693GIn); (B) osmotic HBA2 (chr16: 223678); (C) osmotic HBB (rs334/Gul7Val) (HbS). $n=12,219$ for all osmotic analyses. Oxidative hemolysis: (D) Oxidative SEC14L4 (AX-83171224/rs9606739) Arg112Gly; (E) oxidative G6PD (rs1050828) Val68Met is on the X chromosome; therefore, male and female sample members are displayed separately. $n=10,007$ for all oxidative analyses. Spontaneous hemolysis: (F) Storage TMC8 (rs7208422) Asn306lle. Minor allele homozygotes are in shades of red, heterozygotes in green, and reference allele homozygotes in shades of blue. $n=12,219$ for all storage analyses. For the box-and-whisker plots, the bounds of the box are the 25th and 75th percentiles, the line in the box is the 50 th percentile/median. The whiskers are 1.5 times the interquartile range (25\%-75\%), and black dots are values outside the whiskers. Ancestry groups: AFR, African Americans; EUR, non-Hispanic Whites; EAS, East Asians; SAS, South Asians; CIH, Caribbean Island Hispanics; MCAH, Mexican/Central American Hispanics; OTH, other.

neous storage hemolysis, TMC8 rs7208422 (Asn306Ile, $P<1.23 \times$ $10^{-8}$; Figure $2 \mathrm{~F}$ ) was GWA significant.

Chromosome 8 had 2 nonoverlapping genome-wide significant loci for osmotic hemolysis within ANK1 (Figure 3, A-D). The first locus is centered on rs4737010 (Figure 3A), and the second is $87 \mathrm{~kb}$ away and centered on the NSV rs34664882 (Ala114Val; Figure 3B). PolyPhen2 and SIFT suggested that rs34664882 is deleterious. The SNP appears to have a large quantitative effect on osmotic hemolysis across multiple ancestry groups, accounting for $3.2 \%$ of the variation in osmotic hemolysis in the combined data set. The second GWA-significant locus near ANK1 is centered on rs4737009, which is in the canonical binding motif for the MAZ and STAT5A transcription factors (Supplemental Figure 5). It is likely that both rs34664882 and rs4737009 are independent and functionally consequential mutations for osmotic hemolysis. Conditional GWA showed these loci (rs34664882 and rs4737009) are fully independent and each is genome-wide significant, conditional on the other locus. Additional conditional GWA suggested there may be 2 or more independent loci at SEC14L4 and PIEZO1 (data not shown).

Within G6PD, the rs1050828 Val68Met variant associated with oxidative hemolysis in this study is a common class III variant, also referred to as G6PD A-. Individuals with class III G6PD variants are susceptible to acute hemolytic anemia when their RBCs are exposed to oxidative stress (32). G6PD deficiency is a chromosome X-linked disorder. Figure 2E shows that female A- heterozygotes have intermediate phenotypes for oxidantinduced hemolysis between the female major allele homozygotes and the few $(n=4)$ female A- homozygotes who are sim- ilar to the male A- hemizygotes. This supports the observation that heterozygotes for many disorders potentially have altered or intermediate phenotypes (33).

Pathway and gene-set enrichment analysis (GSEA) identified 3 Bonferroni-corrected significant groups for osmotic hemolysis: spectrin-associated cytoskeleton $\left(P_{B o n}=6.77 \times 10^{-4}\right)$, Steiner erythrocyte membrane genes $\left(P_{\text {Bon }}=2.58 \times 10^{-3}\right)$, and Nikolsky breast cancer 19 p13 amplicon $\left(P_{B o n}=0.028\right)$. For oxidative hemolysis, there were no gene sets significantly enriched after the Bonferroni correction.

Inference of differential expression. MetaXcan (https://github. com/hakyimlab/MetaXcan) was used to infer expression patterns for all genes based on the genotypes that have been identified by GTEx (https://www.gtexportal.org/home/) as expression quantitative trait loci (eQTLs). The inferred gene expression was correlated with spontaneous storage, osmotic, and oxidative hemolysis in the RBC-Omics cohort. Thirteen genes were predicted to be significantly $(P<0.05)$ differentially expressed and significantly $(P<0.05)$ associated with osmotic $(n=11)$ or oxidative $(n=2)$ hemolysis but not spontaneous storage hemolysis ( $n=0$; Table 2$)$. Of these, 10 were situated within one of the genome-wide significant regions, and 2 others were close $(<700 \mathrm{~kb})$. Most of the genes (SLC4A1, SWAP7O, and MFSD2B) found by MetaXcan encode kinases, channels, and metabolic genes whose mechanisms could be affected by changes in gene expression (34-36). MetaXcan did not identify RBC membrane structural genes, such as ANK1 and SPTA1, which is consistent with the previous observations that disease causative variations in genes encoding structural genes tend to be to gain- or loss-of-function mutations, as opposed to changes in gene expression levels (37-39). The most significant SNP in 

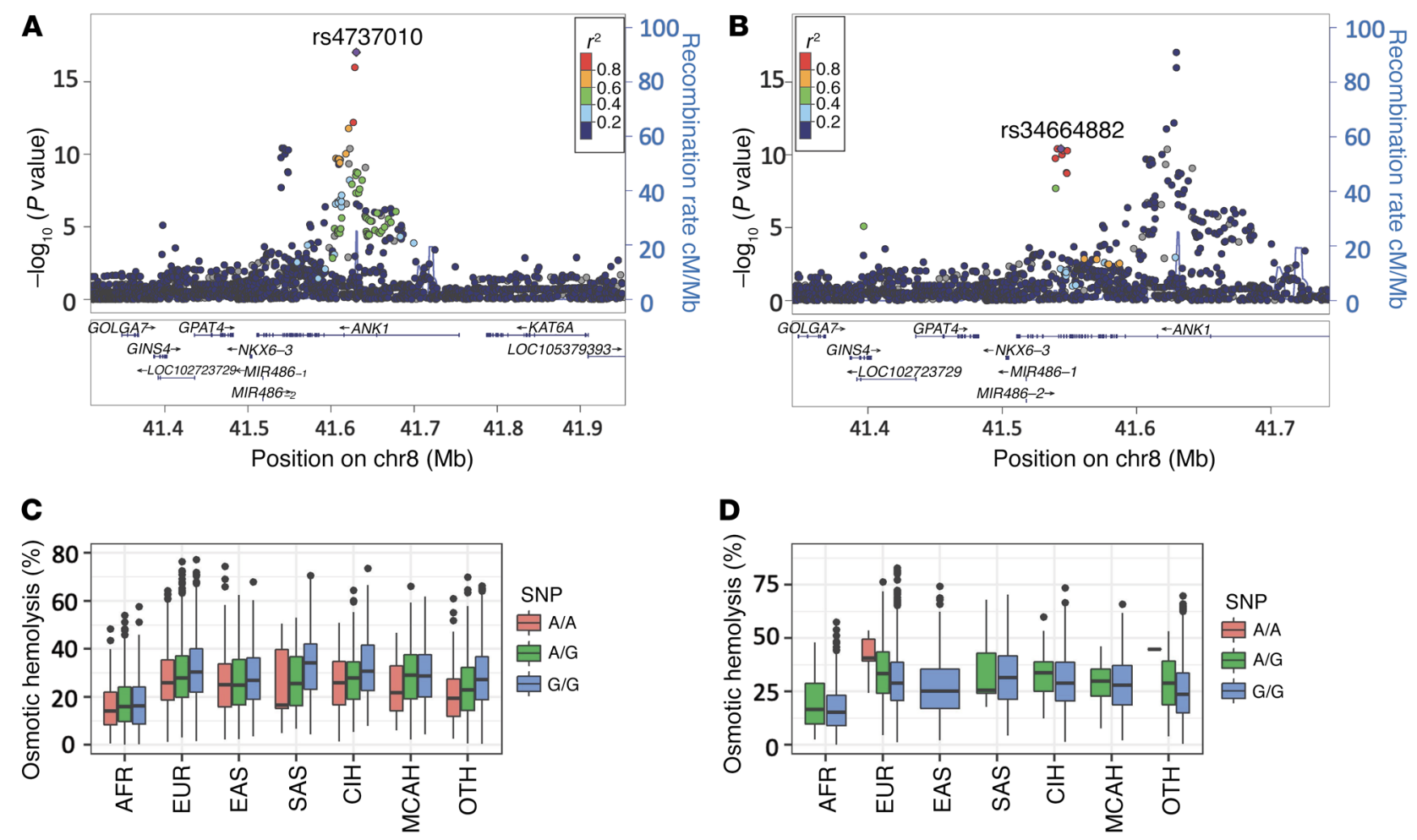

Figure 3. LocusZoom and box-and-whisker plots for $\mathbf{2}$ nonoverlapping genome-wide significant loci in ANK1. (A) LocusZoom plot centered on rs4737010 in ANK1. (B) LocusZoom plot of rs34664882 in ANK1. In these plots, each data point represents an SNP passing quality control in the linear regression analysis of imputed dosage plotted with its $P$ value as a function of genomic position (GRCh38 Assembly). The lead SNP is represented by the purple symbol. The color coding of all other SNPs indicates LD with the lead SNP (estimated by Phase II HapMap CEU $r^{2}$ values): red, $r^{2} \geq 0.8$; gold, $0.6 \leq r^{2}<0.8$; green, $0.4 \leq r^{2}<0.6$; cyan, $0.2 \leq r^{2}<0.4$; blue, $r^{2}<0.2$; gray, $r^{2}$ unknown. Recombination rates are estimated from 1000 Genomes phase 3 data. (C) Box-andwhisker plot of osmotic hemolysis measure by genotype and genetic ancestry group for rs4737010. (D) Box-and-whisker plot of osmotic hemolysis measure by genotype and genetic ancestry group for rs34664882. These figures illustrate 2 nonoverlapping genome-wide significant loci with the ANK1 gene. For the box-and-whisker plots, the bounds of the box are the 25th and 75th percentiles, the line in the box is the 50th percentile/median. The whiskers are 1.5 times the interquartile range (25\%-75\%), and black dots are values outside the whiskers. Ancestry groups: AFR, African Americans; EUR, non-Hispanic Whites; EAS, East Asians; SAS, South Asians; CIH, Caribbean Island Hispanics; MCAH, Mexican/Central American Hispanics; OTH, other.

$\operatorname{GLRX}\left(\mathrm{rs} 72785409 ; P=6.14 \times 10^{-48}\right)$ is an eQTL for GLRX in whole blood based on 15 cohorts in the eQTLGen database (40).

Polygenic scores. We modeled the polygenic scores (PGSs) by using data from two-thirds of the population, whereas data from the remaining third was used for validation. We found the pruning and thresholding model in osmotic hemolysis (at $P<10^{-7}$ and $r^{2}<$ $0.4)$ to validate better than the best LDPred score (correction of best $L D$ pruning $=0.173$ versus best $L D P r e d$ model $=0.0904$; Supplemental Figures 6-9). According to these data for osmotic and oxidative hemolysis, pruning and thresholding is a more precise method of developing PGSs than LDPred.

Table 3 highlights the correlation of each of the 3 hemolysis PGSs within each ancestry group with the observed hemolysis measures. Within non-Hispanic White samples, the correlation with osmotic hemolysis was 0.221 , which explained more of the variability in osmotic hemolysis than any single marker. The best model for oxidative hemolysis was in African American and MCAH samples, where the PGS correlation is approximately 0.260 . Some ancestry groups did not yield PGSs because of small sample sizes or lack of markers with a $P$ value of less than $1 \times 10^{-7}$ when split for cross validation. To develop predictors within these groups, hemolysis measures by ancestry group were correlated with the non-Hispanic White PGS.
This revealed that an ancestry-specific PGS was more precise than those developed in other ancestry groups, even if the latter sample size is larger. Therefore, when possible, PGS should be developed in ancestry-appropriate groups; if not applicable, scores from other ancestry groups can be used but will give diminished precision.

Unlike single-gene disorders in which only a few people contain causal loci, for polygenic traits such as hemolysis everyone has a combination of alleles that increase or decrease hemolysis across all identified loci. For example, for the top 50 loci identified in the non-Hispanic White PGS for osmotic hemolysis, all RBC-Omic donors are heterozygous for between 7 and 34 of the loci (mean $\pm \mathrm{SD}=18.3 \pm 4.6$ ). Thus, genetic factors modulated osmotic and oxidative hemolysis in all individuals.

Genetic analysis of in vivo hemolysis in the WALK-PhASST and PUSH SCD cohorts. To test the hypothesis that the genetic findings obtained from in vitro stress hemolysis perturbations of coldstored RBCs from healthy blood donors may also be relevant to the in vivo severity of steady-state hemolytic anemia in human diseases, the genome-wide significant SNPs identified in the 27 loci for each hemolysis GWA were then tested in 2 cohorts of patients with SCD (Walk-PHaSST and PUSH). Note that there were 232 significant SNPs within these 27 loci. The same SNPs were test- 
Table 2. MetaXcan analysis of genes whose expression is modeled to be associated with osmotic and oxidative hemolysis

\begin{tabular}{|c|c|c|c|c|c|c|c|}
\hline Gene name & Hemolysis & Chromosome location & BF-corrected $P$ value & $r^{2}$ & $q$ value & Number of SNPs & Under GWA Hit? \\
\hline ESYT2 & Osmotic & chr7: 158,523,686-158,622,944 & $4.5 \times 10^{-2}$ & 0.40 & $3.92 \times 10^{-102}$ & 49 & Yes \\
\hline SLC2OA2 & Osmotic & chr8: 42,273,993-42,397,069 & $3.4 \times 10^{-2}$ & 0.23 & $1.28 \times 10^{-53}$ & 24 & No \\
\hline SWAP70 & Osmotic & chr11: 9,685,624-9,774,538 & $1.8 \times 10^{-2}$ & 0.08 & $5.51 \times 10^{-17}$ & 35 & Yes \\
\hline NAA25 & Osmotic & chr12: 112,464,49-112,546,826 & $1.6 \times 10^{-6}$ & 0.03 & $6.09 \times 10^{-7}$ & 17 & Yes \\
\hline FAM109A & Osmotic & chr12: 111,798,455-111,806,925 & $1.0 \times 10^{-3}$ & 0.06 & $1.13 \times 10^{-14}$ & 18 & Yes \\
\hline TMEM116 & Osmotic & chr12: 112,369,086-112,451,023 & $1.4 \times 10^{-2}$ & 0.16 & $3.33 \times 10^{-37}$ & 30 & Yes \\
\hline SLC4A1 & Osmotic & chr17: $42,325,753-42,345,509$ & $9.7 \times 10^{-4}$ & 0.01 & $1.14 \times 10^{-3}$ & 7 & Yes \\
\hline C17orf59 (BORCS6) & Oxidative & chr17: 8,091,651-8,093,564 & $2.7 \times 10^{-2}$ & 0.02 & $4.90 \times 10^{-5}$ & 27 & No \\
\hline GPX4 & Oxidative & chr19: 1,103,936-1,106,787 & $3.2 \times 10^{-6}$ & 0.31 & $8.49 \times 10^{-76}$ & 74 & Yes \\
\hline
\end{tabular}

Presented are the gene name, chromosome location of the gene, Bonferroni-corrected (BF-corrected) $P$ value, predicted performance $\left(r^{2}\right)$ of the models of the gene's expression, predicted performance $q$ value of the model, number of SNPs in the gene used to estimate the gene's expression level, and whether the gene is under one of the genome-wide significant peaks.

ed for association using an in vivo measure of intensity of steadystate hemolytic anemia as a quantitative trait in the SCD patient cohorts. Results between in vitro and in vivo hemolysis were considered consistent if the initial GWA $P$ value was significant at the genome level $\left(P<5 \times 10^{-8}\right)$ and the $P$ value for the association in the 2 SCD cohorts was also significant $(P<0.05)$.

Consistent results were found in 7 regions, including 4 regions for osmotic hemolysis GWA and 3 of 4 regions from the oxidative hemolysis GWA $(P<0.05$; Table 4$)$. Significant results were found for osmotic hemolysis on chromosomes 7 (AQP1), 12 (several genes), and 16 (HBA2, PIEZO1). Oxidative hemolysis was concordant for 3 of the 4 genome-wide significant loci including on chromosome 5 (GLRX), 22 (SEC14L4), and X (G6PD). Even using more conservative assessments, the $H B A 2$ and $G 6 P D$ loci were significant in the SCD cohorts with Bonferroni's testing correction.

\section{Discussion}

This study is the first genome-wide evaluation to our knowledge of in vitro RBC stress hemolysis in cold-stored samples from blood donors, with secondary assessment of GWA-significant findings on the in vivo severity of baseline (steady-state) hemolytic anemia in SCD patients. Increased hemolysis is a hallmark of several diseases, including SCD, and is associated with worse transfusion outcomes, such as poor RBC recovery and increased rates of posttransfusion sepsis. This notion is supported by recent murine studies demonstrating mouse strain-specific susceptibility to $\mathrm{RBC}$ cold-storage injury that correlates with posttransfusion $\mathrm{RBC}$ recovery and function $(4,7,11)$. In addition to limiting storage time and reducing posttransfusion $\mathrm{RBC}$ recovery, hemolysis drives endothelial dysfunction and vascular injury. We and others have demonstrated that cell-free hemoglobin released during hemolysis in the setting of SCD and transfusion of aged stored blood is toxic, driving nitric oxide depletion, oxidative injury, hememediated inflammation, and iron overload (19-21, 23, 24).

We identified 20 loci that were genome-wide significant in All Ancestries sample analysis $\left(P<5 \times 10^{-8}\right)$ for at least one of the hemolysis measures (Table 1). Many of the identified variants were concentrated in proteins known to cause human RBC disorders characterized by RBC fragility such as dehydrated hereditary stomatocytosis (PIEZO1; refs. 41, 42), spherocytosis (ANK1, SPTA1, and SLC4A1; refs. 23, 43), ellipto-poikilocytosis (SPTA1; ref. 44), xerocytosis (PIEZO1; ref. 30), $\alpha$-thalassemia (HBA2; ref. 31 ), and spontaneous and oxidative stress-induced hemolytic anemia (HK1 and G6PD; refs. 16, 45). Providing additional validity, many of the implicated SNPs have been associated with laboratory complete blood cell count measurements, such as reticulocyte counts (SPTA1 and PIEZO1; ref. 46) and other complete blood count indices (G6PD; ref. 47). Consistent with the relevance of our in vitro quantitative measures of stress hemolysis, the identified SNPs from the RBC donor GWAS cohort in $\alpha$-thalassemia, G6PD, PIEZO1, AQP1, SEC14L4, and GLRX were found to GWA-significantly associate with hemoglobin and hemolytic lab indices in the blood of SCD patients.

In addition to genes known to alter RBC function and hemolytic propensity and promote disorders (e.g., spherocytosis and xerocytosis), we identified a number of genes not previously known to impact RBC function, including $M Y O 9 B$. We also identified 7 loci, including $H B B, H B A 2, G 6 P D$, and EYS2, that were genome-wide significant in at least one non-Hispanic White ancestry group (African American, East Asian, South Asian, CIH, $\mathrm{MCAH}$, and Others, which includes multiracial people, Alaska/ Hawaiian/Native Americans, and Pacific Islanders) (Table 1), highlighting the importance of studying diverse populations to provide a more comprehensive evaluation of genetic factors that affect RBC hemolysis. The number of discoveries in the specific ancestry groups is fewer than in the non-Hispanic White population in part due to the lower power from reduced sample sizes in these populations. For some of the loci such as G6PD and $H B B$ in African Americans, the effect is likely due to known variation in these genes such as the A- and HbS variants that are mostly absent in non-Hispanic White populations. This was not always the case; the specific variants identified in MCAH in EYS or rs118149920 


\section{Table 3. Ancestry and cross-ancestry polygenic risk scores}

\begin{tabular}{|c|c|c|c|c|c|}
\hline Genetically defined ancestry ${ }^{A}$ & $\begin{array}{l}\text { Sample } \\
\text { size }\end{array}$ & $\begin{array}{l}\text { Correlation with } \\
\text { osmotic hemolysis }^{B}\end{array}$ & $\begin{array}{l}\text { Correlation with EAU } \\
\text { osmotic PGS }\end{array}$ & $\begin{array}{c}\text { Correlation with } \\
\text { oxidative hemolysis }^{\mathrm{B}}\end{array}$ & $\begin{array}{c}\text { Correlation with EAU } \\
\text { oxidative PGS }\end{array}$ \\
\hline Non-Hispanic White (EAU) & 7,757 & 0.221 & 0.221 & 0.0834 & 0.0834 \\
\hline African American & 1,052 & NA & 0.117 & 0.259 & 0.103 \\
\hline East Asian & 1,112 & 0.180 & 0.134 & NA & 0.0126 \\
\hline South Asian & 265 & NA & 0.184 & NA & 0.125 \\
\hline Caribbean Island Hispanics & 497 & NA & 0.182 & NA & 0.0635 \\
\hline Mexican Central American Hispanics & 459 & 0.251 & 0.184 & 0.263 & 0.0901 \\
\hline Other & 598 & NA & 0.208 & NA & 0.0855 \\
\hline
\end{tabular}

Summary of correlation of polygenic score (PCS) calculated in ancestry-specific groups with each hemolysis measure in the entire ancestry-specific group. AThe ancestry groups are defined by principal component analysis based on genetic data (Figure 1). ${ }^{\mathrm{B}}$ The correlation within ancestry was calculated between the PGSs trained within each ancestry and the measured osmotic or oxidative hemolysis in each ancestry group. NA indicates that no PCS could be calculated that was different from zero. 'The correlation from non-Hispanic White (EAU) was calculated between the PCS trained in this group and the measured osmotic or oxidative hemolysis within each ancestry group.

on chromosome 13 are unlikely to be the causative variants but are likely to be in LD with actual causative variants that could be on chromosomes of Native American ancestry, especially since the $\mathrm{G}$ allele at rs118149920 is absent in European and African populations, but common in Native American and Asian populations.

The validity of the identified regions in the current study of hemolysis in the RBC-Omics cohort is supported by 4 observations: (a) the biological plausibility of the identified SNPs, with most in proteins known to cause RBC disorders such as dehydrated hereditary stomatocytosis (PIEZO1; ref. 41), spherocytosis (ANK1, SPTA1, and SLC4A1; refs. 23, 43), ellipto-poikilocytosis (SPTA1; ref. 44), xerocytosis (PIEZO1; ref. 30), $\alpha$-thalassemia (HBA2; ref. 31 ), and spontaneous and severe nonspherocytic hemolytic anemia (HK1; ref. 45); (b) some of the SNPs have been associated with laboratory complete blood cell count measurements, such as reticulocyte counts (SPTA1 and PIEZO1; ref. 46) and other complete blood count indices (G6PD; ref. 47); (c) MetaXcan (Table 2) finds that the variation in a number of GWA-significant genes contain eQTL for those genes and that the genes' expression are associated with hemolysis measures; and (d) the consistency of GWA findings with significant SNPs that modulate the severity of in vivo hemolysis in patients with SCD (Table 4).

There were a number of variants identified in RBC antioxidative enzymes. For example, the finding that genetic variations in the GPX4 gene modulated oxidative hemolysis is of interest because this enzyme has been linked to key regulatory pathways in erythropoiesis, including erythroblast enucleation and reticulocyte maturation (48-50). With regard to antioxidative activity, GPX4 neutralizes bioactive lipid hydroperoxides to lipid alcohols, thereby preventing iron-dependent cell death, or ferroptosis $(51,52)$. Metabolomics studies of the RBC storage lesion have demonstrated the formation and accumulation of inflammatory bioactive lipids (oxylipins; e.g., 12-hydroxyeicosatetraenoic acid) during cold storage $(53,54)$. Therefore, genetic mutations that compromise RBC GPX4 function may contribute to transfusion-related oxidative injury and inflammatory reactions. There were also significant associations between X-linked G6PD A(the V68M variant) and both in vitro oxidative hemolysis and the in vivo severity of hemolytic anemia in patients with SCD. The enzyme G6PD controls the pentose phosphate pathway-dependent generation of reduced NADPH, necessary for reduction of intracellular glutathione. G6PD A- (V68M) is common in African Americans; approximately $11 \%$ of African American men are hemizygous for this SNP. The enzymatic activity of G6PD A- in erythrocytes is moderately decreased: $10 \%$ to $23 \%$ of normal activity. Hemizygotes do not have chronic hemolysis but can undergo acute hemolysis if exposed to oxidative stress (32). The finding of more marked hemolysis in G6PD A- hemizygous and homozygous SCD persons under basal circumstances in this study would reflect the ongoing oxidative stress that sickle cell erythrocytes experience (55). As mentioned in the Introduction, this variant has also been shown to directly relate to posttransfusion RBC recovery (16), highlighting the potential relevance of our GWA findings to transfusion medicine outcomes.

PGSs were developed for oxidative and osmotic hemolysis in several of the ancestral groups (Table 3) that were able to predict far more of the variance in hemolysis than any one SNP or gene locus alone. The application of non-Hispanic White-developed PGSs to other ancestry groups has enabled the calculation of a PGS when there is not sufficient power to develop an ancestry-specific PGS (56), although the transferability of PGSs across ancestry groups should be viewed with caution (56-58). For example, in African Americans the correlation for PGS with oxidative hemolysis with an ancestry-specific PGS was 0.259, but with the non-Hispanic Whites the PGS was only 0.103. When possible, ancestry-specific PGSs should be developed and used appropriately. PGS will be useful for leveraging the combined genetic effect on individuals and can be combined with other clinical and omics data to gain insights into the pathways leading to RBC function. All individuals in the cohort have some combination of alleles across the loci contribution to the PGS. For the top 50 loci identified in the non-Hispanic White PGS for osmotic hemolysis, none, across all racial groups, contains minor alleles at fewer than 7 of the loci or more than 34 . Thus, genetic variation contributes to variation in oxidative and osmotic hemolysis in all individuals.

We were unable to identify a true replication cohort for the in vitro hemolysis measures of the RBC-Omics cohorts since this is the first such study to our knowledge to explore stress hemoly- 
Table 4. Testing of osmotic, oxidative, and storage hits from the REDS-III RBC-Omics full data in the combined Walk-PHaSST and PUSH cohorts

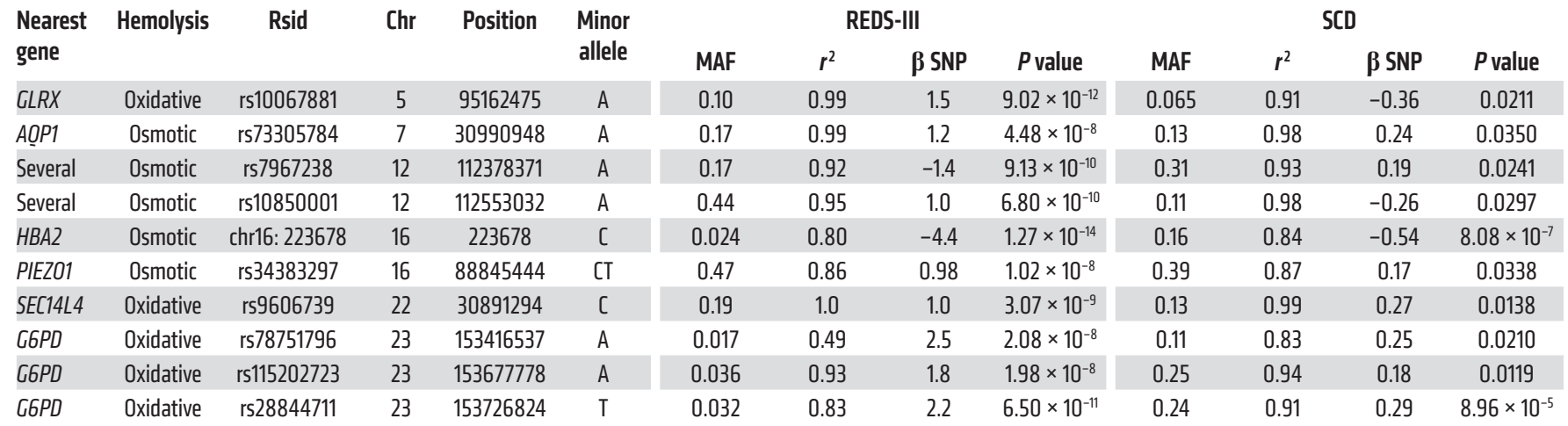

Significant SNPs with nominal $P$ value $<0.05$ in the SCD study were pruned so that linkage disequilibrium $r^{2}<0.3$ in SCD cohorts. Information includes Rsids for markers, the nearest genes, chromosome location, the nucleotide for the minor allele, the minor allele frequency (MAF) from the REDS-III RBC-Omics full data and from the combined Walk-PHaSST and PUSH cohorts, the $r^{2}$ for imputation accuracy, and $P$ value and $\beta$ estimation of the association between minor allele and hemolysis trait. Civen different measures of hemolysis, the directions of the $\beta$ s are not necessarily consistent between the in vivo and in vitro measures. RBC-Omics $n=12,219$ for oxidative hemolysis and 10,017 for osmotic hemolysis. SCD $n=711$ (Walk-PHaSST $n=429 ;$ PUSH $n=282$ ).

sis as a quantitative trait in a large donor population. Instead, we chose to test whether the RBC-Omics results can be generalized to in vivo levels of anemia and hemolysis in the Walk-PHaSST and PUSH SCD cohorts. We found that several variants and regions associated with in vitro hemolysis measures in the REDS-III RBC-Omics donor population were also significant for in vivo hemolysis measures within the SCD WALK-PhASST and PUSH cohorts, such as HBA2, HBB, GLRX, AQP1, and SEC14L4 (Table $4)$. These observations suggest that the in vitro stress measurements identified known and new variants that, under the stress of human disease, may modulate RBC biology. Such findings could lead to identifying rare variants that may modulate the outcomes of many hemolytic diseases. Consistent with this hypothesis, one of the variants identified, G6PD A-, has been recently shown to reduce posttransfusion $\mathrm{RBC}$ recovery (16).

We propose that the identification of genetic variables that modulate the stability of RBCs in storage after response to stressors and the functional integrity of RBCs after transfusion could advance donor selection and storage policies and improve transfusion outcomes. Identification and removal of genetically susceptible "fragile" RBC donors/units that rapidly degrade in storage and selection of profiled "super donor" blood components that might be stable for longer periods of storage or survive longer after transfusion could provide for a precision transfusion medicine strategy, more advanced than current random sampling of donors and transfusion of RBC units irrespective of donor genotypes, recipient disease status, or short- or long-term transfusion requirements.

Further studies are needed to understand the manner in which the genetic variation leads to changes in expression, protein, epigenome, and metabolome, and to understand the interaction network that led to interindividual differences in hemolysis $(59,60)$. For example, we have studied the metabolomic changes induced by the G6PD A- variant associated with oxidative hemolysis and identified significant effects on the NADPH- and glutathione- dependent detoxification pathways of oxidized lipids (61), which could lead to alterations in the dynamics of the RBC membrane.

The genetic information developed in this study is being used in new studies of donor-blood component-recipient outcomes to evaluate the consequences of some of the reported gene variants for transfusion efficacy in patients $(62,63)$. Additional effort is aimed at evaluating nongenetic factors $(64,65)$ that influence RBC-recipient outcomes. Current studies are underway to advance the field of precision transfusion medicine via the development of a transfusion-specific microarray that would provide enhanced tools for the screening of blood donors.

\section{Methods}

RBC-Omics cohort. The REDS-III RBC-Omics cohort donor recruitment and study design are described in detail in Endres-Dighe et al. (25). Briefly, 13,403 whole-blood donors over the age of 18 were recruited from December 2013 to December 2015 at 4 REDS-III blood centers. All subjects were healthy allogeneic blood donors who passed screening and were not anemic. Samples were excluded because of duplicate enrollment, low call rate $(<97 \%)$, sample swap, if blood donation quantity was not sufficient, and if markers of infectious disease were reactive. We analyzed only 1 relative per family, selected based on having the most complete data. The final informative sample size was 12,353. Institutional review board approvals were obtained at all institutions.

Evaluation of donor predisposition to in vitro hemolysis. Stored (3942 days) leukocyte-reduced RBCs were evaluated for spontaneous (cold storage) and 2 stress hemolysis assays including osmotic fragility and oxidative hemolysis using 2 '-azobis(2-amidinopropane) dihydrochloride, as detailed elsewhere $(2,66)$. Each of the hemolysis measures is a quantitative trait on the range from $0 \%$ to $100 \%$ (osmotic mean $=28 \%$, oxidative mean $=36 \%$, and storage $=0.4 \%)(2)$.

Genotyping. Samples were genotyped on a Transfusion Medicine microarray (TM-Array) (26) that contained a total 879,000 SNPs (gen- 
otype coverage of $>90 \%$ for SNPs with MAF $>5 \%$ ) for non-Hispanic White, Hispanics, African Americans, and Asians. The data from this study are available in dbGAP with accession number phs001955.v1.p1 (67). We used PLINK (68) to perform quality control for genotyped data to eliminate potential biases. Individuals for whom calculated genetic sex and self-reported gender differed as well as individuals with more than $3 \%$ missing genetic data were excluded. SNPs with genotype-missing rates higher than $3 \%$ or failing a Hardy-Weinberg equilibrium validation $\left(P<1 \times 10^{-4}\right)$ in any ancestry group were excluded from the study. A total of 811,782 SNPs passed these steps and were used for the imputation.

Imputation. Statistical phasing was conducted by Shape-IT (69). Imputation was then conducted for each 2-Mb interval with 1-Mb flanking regions on each side using Impute2 (70) and 1000 Genomes Project phase 3 as reference haplotypes. Imputation results were further filtered by using an INFO score greater than 0.8 before conducting association analyses (70). The final high-quality data set had 8.1 million SNPs at MAF greater than $5 \%$ and 14 million SNPs at MAF greater than $1 \%$. We ran GWA analysis in all ancestry groups for the 14 million SNPs.

Ancestry. Ancestry analysis was conducted in the RBC-Omics cohort with 1000 Genomes phase 3 samples (Figure 1A and Supplemental figures 1-3). Ancestry principal components (PCs) were calculated using Bioconductor package SNPRelate (71) in the entire data set and separately for participants within each genetic ancestry group. The RBC-Omics subjects were then divided into 7 ancestry groups: non-Hispanic White, African American, East Asian, South Asian, $\mathrm{CIH}, \mathrm{MCAH}$, and Others, which includes multiracial people, Alaska/ Hawaiian/Native Americans, and Pacific Islanders for GWA analyses.

GWA study of common genetic variation. Association analyses were conducted using the software ProbABEL (72). In previous studies, we determined that sex, age, ancestry, and donation frequency were associated with the levels of storage, osmotic, and oxidative hemolysis and were thus were used as covariates as well as the first 10 ancestry PCs in our genetic analyses (2). The distributions of osmotic and oxidative hemolysis were normally distributed, but storage hemolysis was skewed; thus, we used log-transformed storage hemolysis values (Supplemental Figure 10).

We conducted 2 types of analysis: The first was an analysis of all subjects, called ALL Ancestries in the Tables and Figures. In the second, we conducted individual ancestry GWA analyses for non-Hispanic Whites, East Asians, South Asians, CIH, MCAH, and African Americans. A GWA analysis for this study is a multivariable linear model with $P$-value threshold of $5 \times 10^{-8}$ defined as statistically significant for all GWA analyses (Table 1). Conditional GWA analysis verified whether any of the significant loci were independent by incorporating the SNPs with the smallest $P$ value in a region as a covariate in the GWA model and testing the region $50 \mathrm{~kb}$ on each side of this SNP. LD score regression (74) was used to estimate the SNP-based heritability $\left(h^{2}\right)$ of both osmotic and oxidative hemolysis.

Bioinformatic analyses. HaploReg v4.1 (75) was used to annotate the genes nearest to the index SNPs. Version 1.3 of LocusZoom (76) was used, with 1000 Genome phase 3 LD estimation. MetaXcan was implemented to infer gene expression patterns based on genotyped and imputed SNPs from the REDS/RBC-Omics in 922 whole-blood expression profiles from the Depression Gene Network (Table 2 and ref. 71). MetaXcan uses a combination of linear and multivariate linear models with a Bonferroni-corrected (based on number of genes) $P$ value of less than 0.05 being the significance cutoff. FUMA (77) was used to explore the biological pathways and enriched gene set related to osmotic and oxidative hemolysis using the $P$ values from the GWA results for all subject analyses. The curated gene sets and Gene Ontology (GO) terms tested were from the Molecular Signatures Database (MSigDB) in GSEA (78).

PGS for hemolysis. PGSs provide a quantitative metric of the effect of the magnitude of an individual's inherited factors on a trait based on the cumulative impact of many common polymorphisms (79). Several methods for calculating PGS exist including LD pruning, $P$-value thresholding, and LDPred (80), which were applied to calculate PGSs for oxidative, osmotic, and storage hemolysis. Models were built in two-thirds of the samples selected at random without reference for various $P$-value and LD prunes that were validated independently in the remaining third. The two-third/one-third split was chosen as an intermediate of the possible splits suggested by different machine-learning approaches (81). The LD pruning and $P$-value thresholding (Supplemental Figures 6-9) model provided the best estimate, as measured by the correlation between the hemolysis measure in the omitted one-third and the PGS model built in the remaining two-thirds, with LD pruning $r^{2}$ less than 0.2 and $P$-value thresholding at $P$ less than $1 \times 10^{-7}$. This set of thresholds was then used in the entire data set to estimate the final PGS (Table 3). All markers in the final PGS had a $P$ value of less than $1 \times 10^{-7}$.

In vivo studies in WALK-PHaSST and PUSH SCD cohorts. The SNPs that were genome-wide significant from the REDS-III cohort were tested in the Treatment of Pulmonary Hypertension and SCD with Sildenafil Therapy (WALK-PHaSST dbGAP accession PHSO01513. v1.p1) and Pulmonary Hypertension and Hypoxic Response in SCD (PUSH dbGAP accession PHSO01682.v1.p1). This included 232 SNPs in 27 loci common in SCD cohorts (MAF > 0.05) and imputed with $r^{2}$ greater than 0.8 , all with $P$ values less than $5 \times 10^{-8}$ in the REDS-III cohort. We evaluated the association of SNPs with a mathematical measure of the severity of in vivo hemolysis at steady state (baseline, not during a vaso-occlusive event). The endpoint used is the first factor of a previously validated PC measure of severity of steady-state hemolysis in SCD patients (21). The PC is derived from clinically available standard lab measures that reflect RBC hemolysis. These measures include log-transformed serum lactic acid dehydrogenase, aspartate aminotransferase, and total bilirubin, as well as the square root-transformed percentage reticulocytes, and venous hemoglobin levels (21). These measures were adjusted for clinical site of blood collection and were standardized. This estimate of the severity of hemolytic anemia has been previously validated in patients with SCD and shown to significantly correlate with plasma hemoglobin and plasma RBC microparticles, as well as associate with clinical measures that modulate the intensity of hemolysis (HbF level and $\alpha$-thalassemia; refs. 21, 82). Genetic association of the severity of hemolysis by PCA adjusted for age, sex, hemoglobin genotype severity (SS and S- $\beta^{0}$ versus SC and S- $\beta+)$, cohort, use of hydroxyurea validated by HbF level, recent transfusion, and population stratification. Recent transfusion was defined by hemoglobin A level greater than 50\% in Walk-PHaSST and transfusion within the past 2 months in PUSH. Results between in vitro and in vivo hemolysis were considered consistent if the initial GWA $P$ value was significant $\left(P<5 \times 10^{-8}\right)$ and the $P$ value for the association in the 2 SCD cohorts was significant $(P<0.05)$.

The Walk-PHaSST study has 429 analyzable informative patients at least 12 years of age from 9 US Centers and 1 UK Center $(83,84)$. The PUSH study was conducted at 4 tertiary medical centers in the 
United States and contains 282 analyzable patients 3 to 20 years of age (82). These SCD samples were genotyped on the Illumina Human 610-Quad SNP Array, which covers 588,451 genome-wide SNPs. Sample and SNP quality control was described previously (85). Genotypes were phased (85) and imputed (86) to 1000 Genomes phase 3 data using African reference population samples. PCs of autosomal SNPs were estimated using GCTA software (86).

Statistics. Primary GWA analysis was evaluated using 2-sided multivariate linear models, with $P$ values less than $5 \times 10^{-8}$ considered significant. In the SCD cohorts, the replication analysis was a 2-sided multivariable linear model with a $P$ value of less than 0.05 considered replication. MetaXcan analysis was a 2-sided linear model with Bonferroni's correction for the number of genes, with a $P$ value of less than 0.05 considered significant.

Study approval. RBC-Omics was conducted under regulations applicable to all human subject research supported by federal agencies. The Data Coordinating Center (RTI International) of REDS-III was responsible for the overall compliance of human subjects to regulator protocols, including institutional review board approval from each participating blood center, from the REDS-III Central Laboratory (Vitalant) and the Data Coordinating Center. Approval of the WalkPHaSST study protocol (clinical trial NCT00492531) was obtained from local institutional review boards or ethics committees, and written informed consent was obtained from all study subjects in accordance with the Declaration of Helsinki.

\section{Author contributions}

GPP, MPB, YJG, VRG, MCL, AEM, RGC, BRS, JEK, SMED, DB, SK, and MTG conceived of and designed the study. MCL, AEM, RGC, BRS, JEK, SMED, DB, SK, and MTG acquired and prepared data and samples. MTG and TK performed hemolysis and lab assays. GPP, YJG, FF, XZ, and MTG performed statistical analyses. GP, YJG, FF, XZ, MPB, TK, and MTG interpreted data. MN assisted with the analyses related to GWA of the sickle cell disease cohorts. GPP, YJG, MPB, TK, XZ, VRG, and MTG wrote the manuscript. All authors contributed to the critical revision of the manuscript for important intellectual content. GPP and TK are joint first authors with GPP in the lead role because GPP led the writing of the manuscript since he is a geneticist, and the paper is focused on genetics. TK is an expert in hemolysis assays and contributed extensively to the writing.

\section{Acknowledgments}

The authors thank the RBC-Omics research staff at all participating blood centers, the testing laboratories for performing tests and for their contribution to this project, and all blood donors who agreed to participate in this study. The authors also thank Qinzi Xu for his assistance with figure preparation. The NHLBI REDS-III program was supported by NHLBI contracts HHSN2682011-00001I, -00002I, -00003I, -00004I, -00005I, -00006I, -00007I, -00008I, -00009I, 75N2019D00033, and R01 098032. See Supplemental Acknowledgments for NHLBI REDS-III program details.

Address correspondence to: Grier P. Page, RTI International, 2987 Clairmont Rd, Suite 400, Atlanta, Georgia 30340, USA. Phone: 770.407.4907; Email: gpage@rti.org. Or to: Mark T. Gladwin, University of Pittsburgh, 1218 Scaife Hall, 3550 Terrace Street, Pittsburgh, Pennsylvania 15213, USA. Phone: 412.648.9641; Email: gladwinmt@upmc.edu.
1. Tzounakas VL, et al. Donor variation effect on red blood cell storage lesion: a multivariable, yet consistent, story. Transfusion. 2016;56(6):1274-1286.

2. Kanias T, et al. Ethnicity, sex, and age are determinants of red blood cell storage and stress hemolysis: results of the REDS-III RBC-Omics study. Blood Adv. 2017;1(15):1132-1141.

3. Hazegh K, et al. Blood donor obesity is associated with changes in red blood cell metabolism and susceptibility to hemolysis in cold storage and in response to osmotic and oxidative stress. Transfusion. 2021;61(2):435-448.

4. Kanias T, et al. Testosterone-dependent sex differences in red blood cell hemolysis in storage, stress, and disease. Transfusion. 2016;56(10):2571-2583.

5. Chasse M, et al. Effect of blood donor characteristics on transfusion outcomes: a systematic review and meta-analysis. Transfus Med Rev. 2016;30(2):69-80.

6. Chasse M, et al. Association of blood donor age and sex with recipient survival after red blood cell transfusion. JAMA Intern Med. 2016;176(9):1307-1314.

7. Osei-Hwedieh DO, et al. Sickle cell trait increases red blood cell storage hemolysis and post-transfusion clearance in mice. EBioMedicine. 2016;11:239-248.

8. Kanias T, Gladwin MT. Nitric oxide, hemolysis, and the red blood cell storage lesion: interactions between transfusion, donor, and recipient. Trans fusion. 2012;52(7):1388-1392.

9. Lopez C, et al. Mechanisms of genetically-based resistance to malaria. Gene. 2010;467(1-2):1-12.

10. Zimring JC, et al. Strain-specific red blood cell storage, metabolism, and eicosanoid generation in a mouse model. Transfusion. 2014;54(1):137-148.

11. de Wolski K, et al. Metabolic pathways that correlate with post-transfusion circulation of stored murine red blood cells. Haematologica. 2016;101(5):578-586.

12. Kanias T, et al. Frequent blood donations alter susceptibility of red blood cells to storage and stress-induced hemolysis. Transfusion. 2019;59(1):67-78.

13. Lanteri MC, et al. Intradonor reproducibility and changes in hemolytic variables during red blood cell storage: results of recall phase of the REDS-III RBC-Omics study. Transfusion. 2019;59(1):79-88.

14. Francis RO, et al. Frequency of glucose-6-phosphate dehydrogenase-deficient red blood cell units in a metropolitan transfusion service. Transfusion. 2013;53(3):606-611.

15. Raciti PM, et al. Acquired hemoglobin variants and exposure to glucose-6-phosphate dehydrogenase deficient red blood cell units during exchange transfusion for sickle cell disease in a patient requiring antigen-matched blood. J Clin Apher. 2013;28(4):325-329.
16. Francis RO, et al. Donor glucose-6-phosphate dehydrogenase deficiency decreases blood quality for transfusion. J Clin Invest. 2020;130(5):2270-2285.

17. Dern RJ, et al. Studies on the preservation of human blood. I. Variability in erythrocyte storage characteristics among healthy donors. J Lab Clin Med.1966;67(6):955-965.

18. Gordeuk VR, et al. The CYB5R3(c) $(.350 \mathrm{C}>\mathrm{G})$ and G6PD A alleles modify severity of anemia in malaria and sickle cell disease. Am J Hematol. 2020;95(11):1269-1279.

19. Kato GJ, et al. Lactate dehydrogenase and hemolysis in sickle cell disease. Blood. 2013;122(6):1091-1092.

20. Milton JN, et al. Genetic determinants of haemolysis in sickle cell anaemia. Br J Haematol. 2013;161(2):270-278.

21. Nouraie M, et al. The relationship between the severity of hemolysis, clinical manifestations and risk of death in 415 patients with sickle cell anemia in the US and Europe. Haematologica. 2013;98(3):464-472.

22. Saraf SL, et al. Associations of $\alpha$-thalassemia and BCL11A with stroke in Nigerian, United States, and United Kingdom sickle cell anemia cohorts. Blood Adv. 2017;1(11):693-698.

23. Kato GJ, et al. Intravascular hemolysis and the pathophysiology of sickle cell disease. JClin Invest. 2017;127(3):750-760. 
24. Donadee C, et al. Nitric oxide scavenging by red blood cell microparticles and cell-free hemoglobin as a mechanism for the red cell storage lesion. Circulation. 2011;124(4):465-476.

25. Endres-Dighe SM, et al. Blood, sweat, and tears: red blood cell-omics study objectives, design, and recruitment activities. Transfusion. 2019;59(1):46-56.

26. Guo Y, et al. Development and evaluation of a transfusion medicine genome wide genotyping array. Transfusion. 2019;59(1):101-111.

27. Moreno-Estrada A, et al. Human genetics. The genetics of Mexico recapitulates Native American substructure and affects biomedical traits. Science. 2014;344(6189):1280-1285.

28. Nelson SC, et al. Improved imputation accuracy in Hispanic/Latino populations with larger and more diverse reference panels: applications in the Hispanic Community Health Study/ Study of Latinos (HCHS/SOL). Hum Mol Genet. 2016;25(15):3245-3254.

29. van der Harst P, et al. Seventy-five genetic loci influencing the human red blood cell. Nature. 2012;492(7429):369-375.

30. Fortugno C, et al. Hereditary red blood cell membrane defects. Detection of PIEZO1 mutations associated with SPTA1 mutations. An unusual clinical case of hereditary xerocytosis. Pediatr Hematol Oncol. 2021;38(2):184-190.

31. Tatu T, Sweatman D. Hemolysis area: a new parameter of erythrocyte osmotic fragility for screening of thalassemia trait. J Lab Physicians. 2018;10(2):214-220.

32. Luzzatto L, et al. Glucose-6-phosphate dehydrogenase deficiency. Blood. 2020;136(11):1225-1240.

33. Vogel F. Clinical consequences of heterozygosity for autosomal-recessive diseases. Clin Genet. 1984;25(5):381-415.

34. Niss O, et al. Genotype-phenotype correlations in hereditary elliptocytosis and hereditary pyropoikilocytosis. Blood Cells Mol Dis. 2016;61:4-9.

35. Ripich T, Jessberger R. SWAP-70 regulates erythropoiesis by controlling $\alpha 4$ integrin. Haematologica. 2011;96(12):1743-1752.

36. Ferru E, et al. Regulation of membranecytoskeletal interactions by tyrosine phosphorylation of erythrocyte band 3. Blood. 2011;117(22):5998-6006.

37. Hellwege JN, et al. A multi-stage genome-wide association study of uterine fibroids in African Americans. Hum Genet. 2017;136(10):1363-1373.

38. Gao G, et al. Trans-ethnic predicted expression genome-wide association analysis identifies a gene for estrogen receptor-negative breast cancer. PLoS Genet. 2017;13(9):e1006727.

39. Fryett JJ, et al. Comparison of methods for transcriptome imputation through application to two common complex diseases. Eur J Hum Genet. 2018;26(11):1658-1667.

40. Võsa U, et al. Unraveling the polygenic architecture of complex traits using blood eQTL metaanalysis [preprint]. https://doi.org/10.1101/447367. Posted on bioRxiv October 19, 2018.

41. Zama D, et al. A novel PIEZO1 mutation in a patient with dehydrated hereditary stomatocytosis: a case report and a brief review of literature. Ital J Pediatr. 2020;46(1):102.

42. Ma S, et al. A role of PIEZO1 in iron metabolism in mice and humans. Cell. 2021;184(4):969-982.

43. Wang X, et al. Genetic and clinical characteristics of patients with hereditary spherocytosis in Hubei Province of China. Front Genet. 2020;11:953.

44. Iolascon A, et al. A genomic deletion causes truncation of $\alpha$-spectrin and ellipto-poikilocytosis. Blood Cells Mol Dis. 2011;46(3):195-200.

45. Jamwal M, et al. A nonsense variant in the hexokinase 1 gene (HK1) causing severe non-spherocytic haemolytic anaemia: genetic analysis exemplifies ambiguity due to multiple Isoforms. Br J Haematol. 2019;186(5):e142-e145.

46. Astle WJ, et al. The allelic landscape of human blood cell trait variation and links to common complex disease. Cell. 2016;167(5):1415-1429.

47. Ding K, et al. Genetic variants that confer resistance to malaria are associated with red blood cell traits in African-Americans: an electronic medical record-based genome-wide association study. G3 (Bethesda). 2013;3(7):1061-1068.

48. Canli O, et al. Glutathione peroxidase 4 prevents necroptosis in mouse erythroid precursors. Blood. 2016;127(1):139-148.

49. Altamura S, et al. Glutathione peroxidase 4 and vitamin $\mathrm{E}$ control reticulocyte maturation, stress erythropoiesis and iron homeostasis. Haematologica. 2020;105(4):937-950.

50. Ouled-Haddou $\mathrm{H}$, et al. A new role of glutathione peroxidase 4 during human erythroblast enucleation. Blood Adv. 2020;4(22):5666-5680.

51. Forcina GC, Dixon SJ. GPX4 at the crossroads of lipid homeostasis and ferroptosis. Proteomics. 2019;19(18):e1800311.

52. Seibt TM, et al. Role of GPX 4 in ferroptosis and its pharmacological implication. Free Radic Biol Med. 2019;133:144-152.

53. D'Alessandro A, et al. Effects of aged stored autologous red blood cells on human plasma metabolome. Blood Adv. 2019;3(6):884-896.

54. Fu X, et al. Bioactive lipids accumulate in stored red blood cells despite leukoreduction: a targeted metabolomics study. Transfusion. 2016;56(10):2560-2570.

55. Nader E, et al. The red blood cell-inflammation vicious circle in sickle cell disease. Front Immunol. 2020;11:454.

56. Wojcik GL, et al. Genetic analyses of diverse populations improves discovery for complex traits. Nature. 2019;570(7762):514-518.

57. Martin AR, et al. Human demographic history impacts genetic risk prediction across diverse populations. Am J Hum Genet. 2017;100(4):635-649.

58. Cavazos TB, Witte JS. Inclusion of variants discovered from diverse populations improves polygenic risk score transferability. HGG Adv. 2021;2(1):100017.

59. Leopold JA, et al. The application of big data to cardiovascular disease: paths to precision medicine. J Clin Invest. 2020;130(1):29-38.

60. Maron BA, et al. A global network for network medicine. NPJ Syst Biol Appl. 2020;6(1):29.

61. D'Alessandro A, et al. Donor sex, age and ethnicity impact stored red blood cell antioxidant metabolism through mechanisms in part explained by glucose 6-phosphate dehydrogenase levels and activity. Haematologica. 2021;106(5):1290-1302.
62. Roubinian N, et al. Methodological considerations for linked blood donor-component-recipient analyses in transfusion medicine research. ISBT Sci Ser. 2020;15(1):185-193.

63. Roubinian NH, Kanias T. Blood donor component-recipient linkages: is there fire where there is smoke? Transfusion. 2019;59(8):2485-2488.

64. Roubinian NH, et al. Effect of donor, component, and recipient characteristics on hemoglobin increments following red blood cell transfusion. Blood. 2019;134(13):1003-1013.

65. Edgren G, et al. Association of blood donor sex and prior pregnancy with mortality among red blood cell transfusion recipients. JAMA. 2019;321(22):2183-2192.

66. Stone $\mathrm{M}$, et al. Piloting and implementation of quality assessment and quality control procedures in RBC-Omics: a large multi-center study of red blood cell hemolysis during storage. Transfusion. 2019;59(1):57-66.

67. National Institutes of Health. NHLBI Recipient Epidemiology Donor Evaluation Study (REDS)III-Red Blood Cell Omics (RBC-Omics) Study. dbGaP Study Accession: phs001955.v1.p1 https:// www.ncbi.nlm.nih.gov/projects/gap/cgi-bin/ study.cgi?study_id=phs001955.v1.p1. Updated September 4, 2020. Accessed February 7, 2021

68. Purcell S, et al. PLINK: a tool set for whole-genome association and population-based linkage analyses. Am J Hum Genet. 2007;81(3):559-575.

69. Delaneau O, et al. Shape-IT: new rapid and accurate algorithm for haplotype inference. $B M C$ Bioinformatics. 2008;9:540.

70. Howie B, et al. Genotype imputation with thousands of genomes. G3 (Bethesda). 2011;1(6):457-470.

71. Zheng $X$, et al. A high-performance computing toolset for relatedness and principal component analysis of SNP data. Bioinformatics. 2012;28(24):3326-3328.

72. Aulchenko YS, et al. ProbABEL package for genome-wide association analysis of imputed data. BMC Bioinformatics. 2010;11:134.

73. Price AL, et al. Principal components analysis corrects for stratification in genome-wide association studies. Nat Genet. 2006;38(8):904-909.

74. Bulik-Sullivan BK, et al. LD Score regression distinguishes confounding from polygenicity in genome-wide association studies. Nat Genet. 2015;47(3):291-295.

75. Ward LD, Kellis M. HaploReg: a resource for exploring chromatin states, conservation, and regulatory motif alterations within sets of genetically linked variants. Nucleic Acids Res. 2012;40(D1):D930-D934.

76. Pruim RJ, et al. LocusZoom: regional visualization of genome-wide association scan results. Bioinformatics. 2010;26(18):2336-2337.

77. Watanabe $\mathrm{K}$, et al. Functional mapping and annotation of genetic associations with FUMA. Nat Commun. 2017;8(1):1826.

78. GSEA. Molecular Signatures Database v7.2. https://www.gsea-msigdb.org/gsea/msigdb/ index.jsp. Updated April 1, 2021. Accessed May 14, 2021.

79. Torkamani A, et al. The personal and clinical utility of polygenic risk scores. Nat Rev Genet. 2018;19(9):581-590. 
80. Khera AV, et al. Genome-wide polygenic scores for common diseases identify individuals with risk equivalent to monogenic mutations. Nat Genet. 2018;50(9):1219-1224.

81. Kuhn M, Johnson K, eds. Applied Predictive Modeling. Springer; 2013.

82. Minniti CP, et al. Elevated tricuspid regurgitant jet velocity in children and adolescents with sickle cell disease: association with hemolysis and hemoglobin oxygen desaturation. Haematologica.
2009;94(3):340-347.

83. Sachdev V, et al. Echocardiographic markers of elevated pulmonary pressure and left ventricular diastolic dysfunction are associated with exercise intolerance in adults and adolescents with homozygous sickle cell anemia in the United States and United Kingdom. Circulation. 2011;124(13):1452-1460.

84. Machado RF, et al. Hospitalization for pain in patients with sickle cell disease treated with sildenafil for elevated TRV and low exercise capacity. Blood. 2011;118(4):855-864.

85. Zhang $X$, et al. A genetic variation associated with plasma erythropoietin and a non-coding transcript of PRKAR1A in sickle cell disease. Hum Mol Genet. 2016;25(20):4601-4609.

86. Howie BN, et al. A flexible and accurate genotype imputation method for the next generation of genome-wide association studies. PLoS Genet. 2009;5(6):e1000529. 